\title{
Colour Reconnections and Rapidity Gaps
}

\author{
Leif Lönnblad, NORDITA, Blegdamsvej 17, DK-2100 København Ø, Denmark, leif@nordita.dk
}

The models recently proposed by Buchmüller [1] and Ingelman [2] to describe rapidity gap events in DIS at HERA are both based on the assumption of additional colour exchange between the struck system and the proton remnant in boson-gluon fusion events. In this way the struck system may become colour singlet and a rapidity gap may be formed between it and the remnant. In both models, the colour exchange mechanism is assumed to be non-perturbative with negligible momentum transfer. It is clear that such non-perturbative interactions take place very late in the process, and cannot influence the perturbative emissions in an event. Therefore the models rely heavily on the assumption that the emission of hard gluons in the forward region is suppressed, since any such emission would destroy a potential gap.

Naively one may think that additional gluon radiation is suppressed by a power of $\alpha_{S}$, and indeed, the model of ref. [1] does not even consider it. However, the factor $\alpha_{S}$ is more than compensated by the increase of phase space for such emissions at small $x$, introducing large logarithms of $1 / x$. In the model of ref. [2], additional perturbative gluon emissions are taken into account using a parton shower scenario based on the Altarelli-Parisi or DGLAP [3] evolution as implemented in the LEPTO event generator program [if. With this model the rate and distribution of rapidity gap events can be reproduced, provided the parton shower is cut off at a virtuality scale around $4 \mathrm{GeV}^{2}$.

There are serious doubts whether at small $x$, the DGLAP evolution, although being able to describe inclusive distributions like $F_{2}$, is appropriate for the description of exclusive final state properties, such as gluon emission. In the parton-shower language, the problem is that the initial-state shower is strictly ordered in virtuality or transverse momentum $p_{\perp}$. I.e. starting from the electro-weak vertex, tracing successive emissions upwards in $\mathrm{x}$ towards the remnant in rapidity, the $p_{\perp}$ decreases in each step as indicated in fig. 11a. At small $x$ and $Q^{2}$, where the bulk of events are found at HERA, this means that the initial-state shower quickly runs out of virtuality, preventing it from populating the forward region in phase space. Consequently rapidity gaps occur frequently on the partonic level, which may then survive the hadronization by introducing the "Soft Colour Interaction" (SCI) of ref. [2].

There is no a priori reason why the emissions should be strongly ordered. Indeed, in the BFKL [5] and CCFM [6] evolution schemes, where large logarithms of $1 / x$ are resummed, which are believed to be more appropriate in the small- $x$ region, the transverse momenta are unordered. Unfortunately, there is currently no event generator implementing any of these schemes available. There is, however, one generator - ARIADNE [7] - which implements an unordered cascade based on the Dipole Cascade Model (DCM) [8]. Recently, colour rearrangements of produced partons was implemented in this program, introducing the possibility to get large rapidity gaps in a similar way as in the models above, but with an unordered cascade [9].

The difference between the ARIADNE and LEPTO programs is apparent in fig. 1 $\mathrm{b}$, where the rapidity distribution of gluons is shown for the two models. Clearly, ARIADNE gives much more gluons in the forward region. This is reflected in the $\eta_{\max }$ distribution in fig. 2a, where the DCM with reconnections is far below.

But even if the number of gap events could be increased in ARIADne by simply increasing the cutoff in the cascade, thus reducing the number of gluons, distributions such as the mass of the diffractive system $-m_{X}$, would
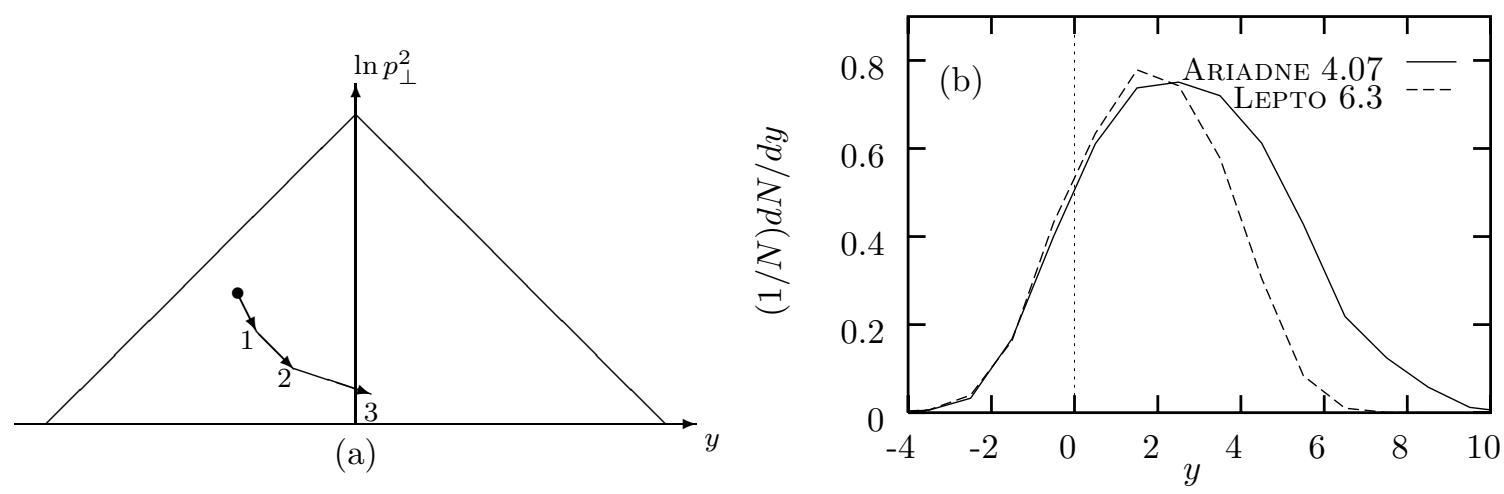

Figure 1: (a) The available phase space available for emission of a gluon with rapidity $y$ and transverse momentum $P_{\perp}$. (b) The rapidity distribution of gluons in the lab frame of HERA. 
(a)

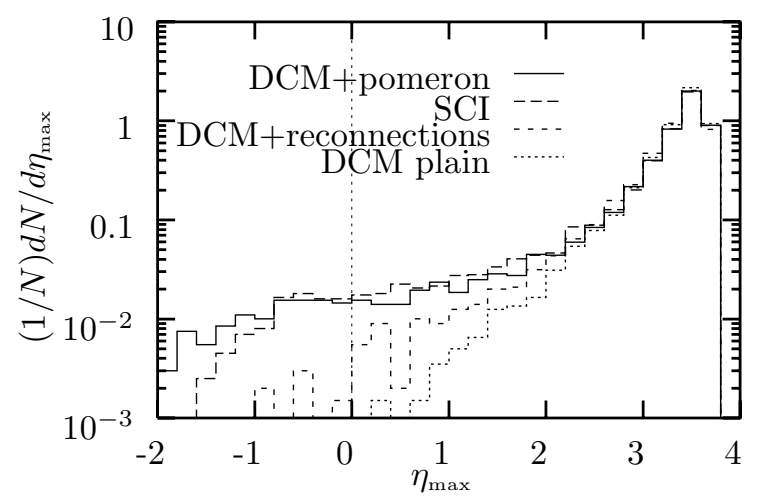

(b)

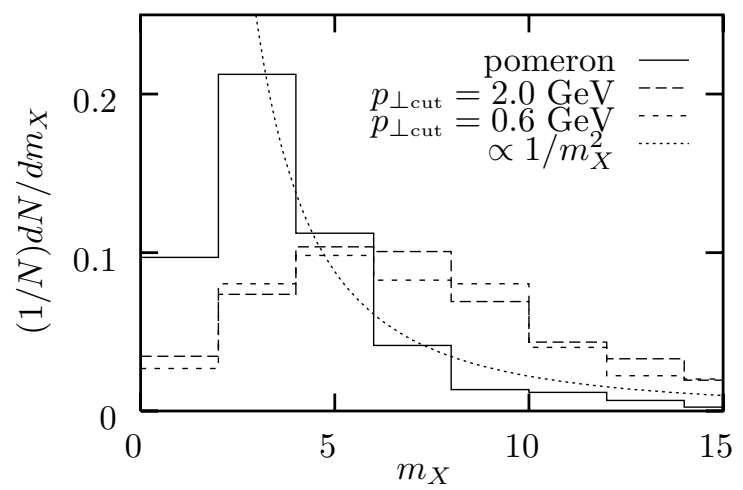

Figure 2: (a) The distribution of $\eta_{\max }$, the largest pseudo-rapidity of any particle in the lab frame of a HERA detector. The full line is ARIADNE with the default pomeron inspired model, long-dashed is SCI, short-dashed is DCM with colour reconnections (CR) and the dotted line is plain DCM without CR or pomerons. (b) The distribution of $m_{X}$ for events at HERA with $\eta_{\max }<3.2$. The full line is ARIADNE with pomerons, long-dashed $D C M$ with CR and an increased cutoff, and short-dashed is the same with default cutoff.

not be well described by ARIADNE because of the different distribution of gluons. This is clear from fig. 2 $\mathrm{b}$ where the $m_{X}$ distribution is shown for two different cutoffs and compared to the results from a pomeron-inspired model 10 which have the $1 / m_{X}^{2}$ behavior seen in data.

In conclusion, the models [1, 2] which describe the rapidity-gap events found in DIS at HERA, do so because they inhibit gluon radiation in the forward region. There is no physical motivation why this should be the done. Using an unordered cascade, where the effect of the increase of available phase space with $\log 1 / x$ is taken into account, give qualitatively different distributions in possible rapidity-gap events and can only explain data using a pomeron inspired model, where the forward radiation is inhibited because of the initial colour structure of the target proton. The models [1, 2] are also based on the assumption of having colour exchanged without exchanging momentum. Note that this exchange means that a charge going very forward in rapidity is suddenly "infinitely decelerated" to go backward in rapidity. It is questionable whether such an acceleration of a charge is allowed without having accompanying radiation, which would both require a momentum transfer, and most likely fill up the potential rapidity gap.

\section{References}

[1] W. Buchmüller, A. Hebecker, Phys. Lett. B355 (1995) 573.

[2] A. Edin, G. Ingelman, J. Rathsman, DESY 95-145, July 1995.

[3] V.N. Gribov, L.N. Lipatov, Sov. J. Phys. 15 (1972) 438 and 675;

L.N. Lipatov, Sov. J. Phys. 20 (1975) 94;

G. Altarelli, G. Parisi, Nucl. Phys. B126 (1977) 298;

Yu.L. Dokshitser, Sov. Phys. JETP 46 (1977) 641.

[4] G. Ingelman, LePTO version 6.3, in Physics at HERA, eds, W. Buchmüller, G. Ingelman, vol. 3, p. 1366, DESY, 1991.

[5] V.S. Fadin, E.A. Kuraev, L.N. Lipatov, Sov. Phys. JetP 45 (1977) 199;

Ya.Ya. Balitsky, L.N. Lipatov, Sov. J. Nucl. Phys. 28 (1978) 822.

[6] M. Ciafaloni, Nucl. Phys. B296 (1988) 49;

S. Catani, F. Fiorani, G. Marchesini, Phys. Lett. B234 (1990) 339; Nucl. Phys. B336 (1990) 18.

[7] L. Lönnblad, Ariadne version 4.07 program and manual, Comput. Phys. Commun. 71 (1992) 15.

[8] G. Gustafson, Phys. Lett. B175 (1986) 453;

G. Gustafson, U. Pettersson, Nucl. Phys. B306 (1988) 746;

B. Andersson et Al., Z. Phys. C43 (1989) 625.

[9] L. LÖnnblad, CERN-TH/95-218 (to appear in Z. Phys. C), August 1995.

[10] L. Lönnblad, Z. Phys C65 (1995) 285. 